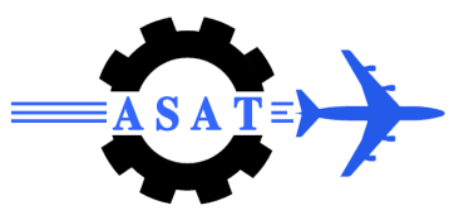

\title{
Preliminary Design and Implementation of a Telemetry and Telecommand Subsystem in Micro-Satellites: An Overview
}

\author{
H.H. El-Banna ${ }^{*}$, A.A. Mitkees ${ }^{\dagger}$, M.M. Mokhtar ${ }^{\ddagger}$, A.M. Allam ${ }^{\S}$
}

\begin{abstract}
A preliminary design and implementation of a Telemetry and Telecommand (T\&Tc) subsystem is presented by using Commercial Off The Shelf (COTS) modules. Telemetry data are collected, stored, and then transmitted to the Ground Station (GS). Commands are executed in both autonomous and GS controlling modes. Commands are executed on the On Board Computer (OBC) according to prepared scheduled commands and patch files. Updated patch files can be loaded from the Ground Control Center (GCC) to the OBC.
\end{abstract}

Keywords: Micro Satellites, Telemetry and telecommand subsystem, embedded systems, FPGA

\section{Introduction}

The satellite mainly consists of integrated subsystems [1]. These main subsystems of a generic satellite are: Attitude determination and control, thermal control, structure and mechanics, power, payload, onboard computer, and telemetry and telecommand subsystem [2].each subsystem has its own components and modules which support the objective of this subsystem, as shown in Fig. (1). T\&Tc subsystem is the means of monitoring, controlling the satellite operations, and communicating with the GS [3]. Telemetry makes: data collection from the other subsystems, process the data into a desired form, data storage, data modulation and transmission, and ranging beacon transmission. Commands from the GCC are received, demodulated, decoded, verified and distributed to the appropriate subsystem. Autonomous control from the satellite's OBC should be obtained. OBC should support different types of data interfaces (Analog, digital, and serial ports).

In this paper, a preliminary design and implementation of a $T \& T c$ subsystem is presented by using COTS modules. Telemetry data are collected, stored, and then transmitted during the access time to the GS according to commands from the OBC. These commands are in both autonomous and GS controlling modes. Commands are executed on the OBC according to prepared scheduled commands and patch files. Updated patch files can be loaded from the $\mathrm{GCC}$ to the $\mathrm{OBC}$.

\footnotetext{
*Egyptian Armed Forces, elbanna3000@yahoo.com

† Egyptian Armed Forces, aamitkees@yahoo.com

Prof. Dr., NARSS

$\S$ Prof. Dr., GUC, allam72000@yahoo.com
} 


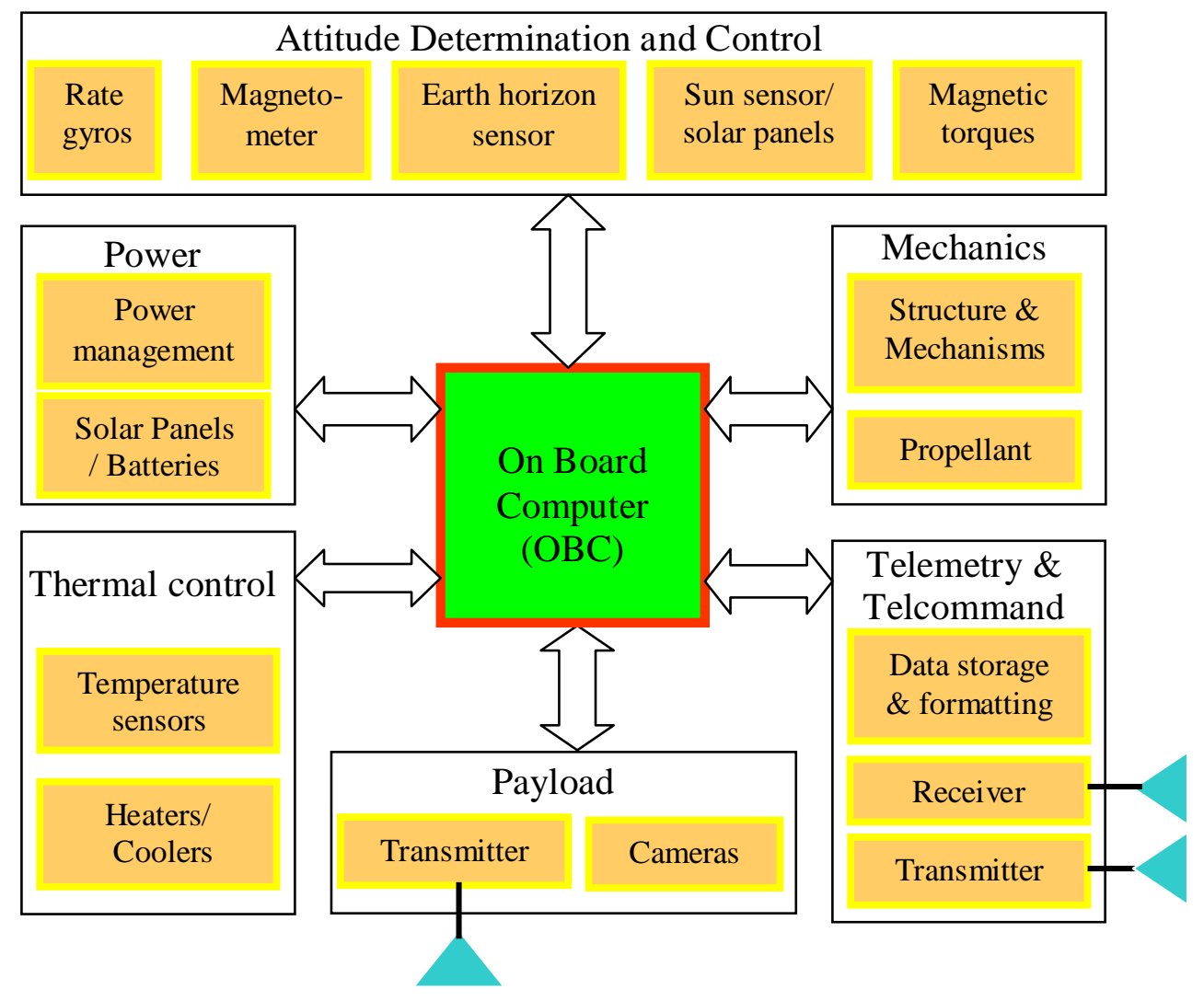

Fig. (1) Top level diagram of a generic satellite

In the past, these subsystems were integrated together as trays and many wires connect between these different modules. These wires may reduce the reliability of the satellite. In these days, updated technology are used, these subsystems are stacked together through PC104 interface [4], and are completely fitted by four screws in the corners of the modules, as shown in Fig. (2) [5].

Most satellites use a computer system to perform control operations and process different types of information and commands. OBC is the brain of the satellite, T\&Tc subsystem is considered as the interface between the GS and the other satellite subsystems.

OBC software may be classified in one of two categories: individual elements of software to run different processes, and the complete operating system (OS). The operating system itself does not directly provide any spacecraft function or directly service any onboard system. Rather, it facilitates the operation of the computer system in much the same way that the spacecraft bus facilitates the operation of the spacecraft.

In this preliminary design, the COTS OBC is the Arcom SBC-GX1 board [6], it is a high performance, high-functionality multimedia PC compatible processor board designed to work in embedded systems. It contains all the standard features found in a PC system with some embedded additions. The used Real Time Operating System (RTOS) is Windows XP Embedded [7]; which is reliable, light and it takes a small size on the compact flash memory of the OBC. Windows XP Embedded facilitates many programming operations, commands, patch files, schedules, sending and receiving of data and commands. It is also accepting to the adding of new components on it. Generation of a new OS image is available and can be transferred and loaded to the satellite while it is on the space. 


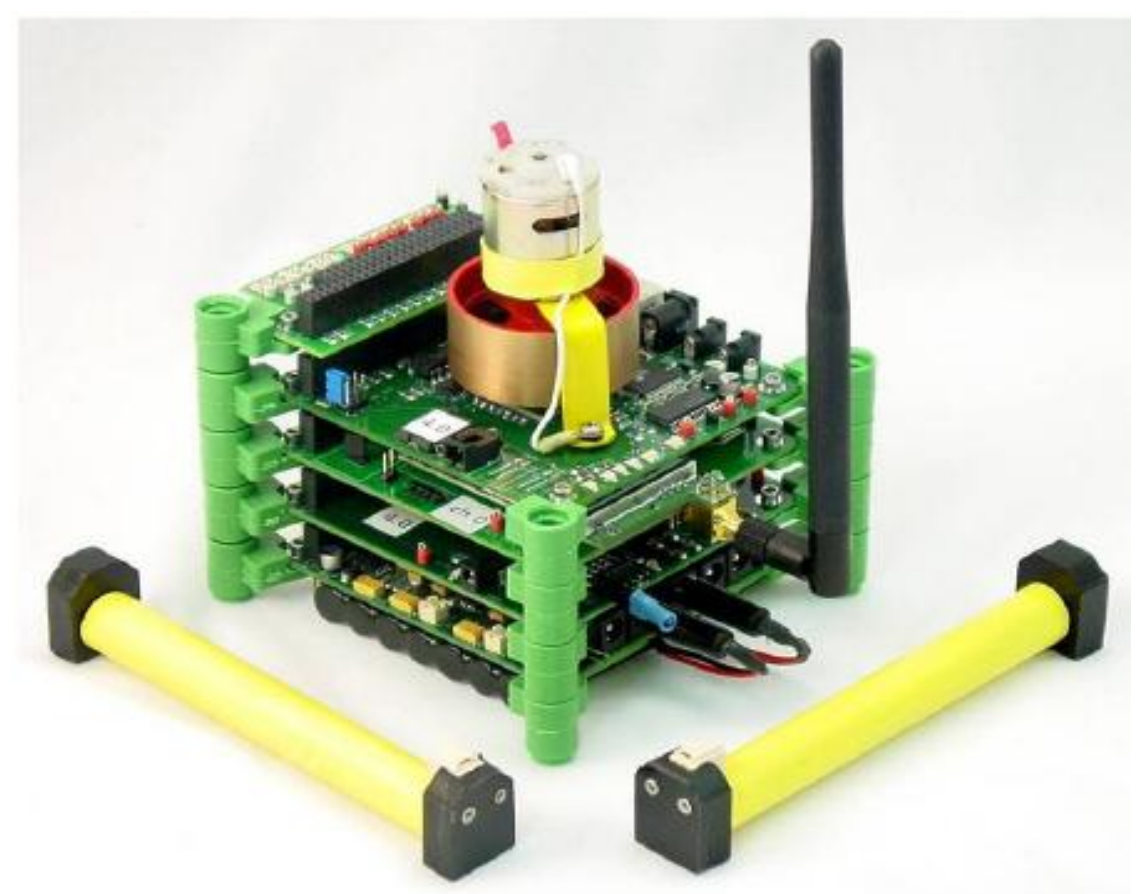

Fig. (2) Example of a complete nano-satellite based on PC-104 bus

The PF3100 ${ }^{\mathrm{TM}}$ FPGA PC/104-plus Module [8], as shown in Fig. (3) is used as a telemetry board, which collects telemetry data from its several input/output ports. This board is connected directly to the OBC via PC/104 connector; which supports all ISA bus signals, it is an ultra high-density reconfigurable module. It uses a Xilinx Virtex-II [9] Platform FPGA (Field Programmable Gate Array). The FPGA can be configured via JTAG, ISA connector, or from flash memory which is built in this module.

\section{Generic Subsystem Data Flow}

The flow of data through the telemetry system begins with the data acquisition system (DAS). The input to the DAS is physical stimuli sampled across multiple channels, and the output is a single digitized data stream. Some examples of physical parameters of interest include temperature, pressure, force, position, and acceleration. The physical stimulus is sensed with either an analog (transducer) or a digital sensor. The transducer translates the physical parameter into an electrical analog, usually in the form of a voltage or current. A signal conditioner provides the electrical gain that drives the system. Usually, signal processing also takes place (such as filtering noise from the data) to accommodate the next step, the measuring device. The next part of the telemetry system is data construction in frames or packets. Then these data are formatted. The purpose of the formatter is to change the format of the packet data stream into the baseband signal delivered to the communication system transmitter [10]. The on-board communication system modulates the baseband signal onto the carrier wave and converts this signal to the transmit frequency. The signal is then amplified and radiated out the transmitter antenna. This process of sending data over a RF link is sometimes called band-pass or modulated transmission. The data flow through the command system is nearly the reverse of the data flow through the telemetry system discussed previously. A Graphical User Interface (GUI) with the GCC generally is used to generate the appropriate commands. In the next sections, some details on the preliminary design of the T\&Tc subsystem are presented. 


\section{Telemetry Design}

The status and conditions of the active components and resources of satellite subsystems are collected from special sensors distributed throughout the satellite. This data is then transmitted periodically or upon a command to the GCC by the telemetry subsystem.

Figure (4) shows the top level diagram of the preliminary design on the telemetry board, it should do main three functions: (1) Collect scientific and hou sekeeping telemetry data and organize it into a desired Pulse Code Modulation (PCM) Matrix, as shown in Fig. (5). Each row of the matrix is called a frame, and each cell represents a single byte. The two bytes at the beginning of a frame forms a synchronization (sync) word, so that the sequence finder can detect the beginning of each frame. The third byte of the frame is the sub-frame identification (SFID), this byte is used to identify the contents of each frame. The PCM matrix can be organized by software or by FPGA design. (2) Storing the telemetry data on a memory location, in this design the FPGA block RAMs are used to store the telemetry data in it, the write enable of this block RAM is activated from the OBC (IOW) signal. Storing of telemetry data is scheduled and a patch file controls the telemetry data storing process. (3) Downlinking the telemetry data according to a prescheduled algorithm or according to a specific transmission command from the GS. Downlinking should be done during the visibility period (visibility between the satellite and the GS), so a patch file was done to excite the downlinking process by using (Input Output Read) IOR control signal from OBC. The telemetry data is then serialized and sent through RS 232 port to the transmitter to the antenna to the GS.

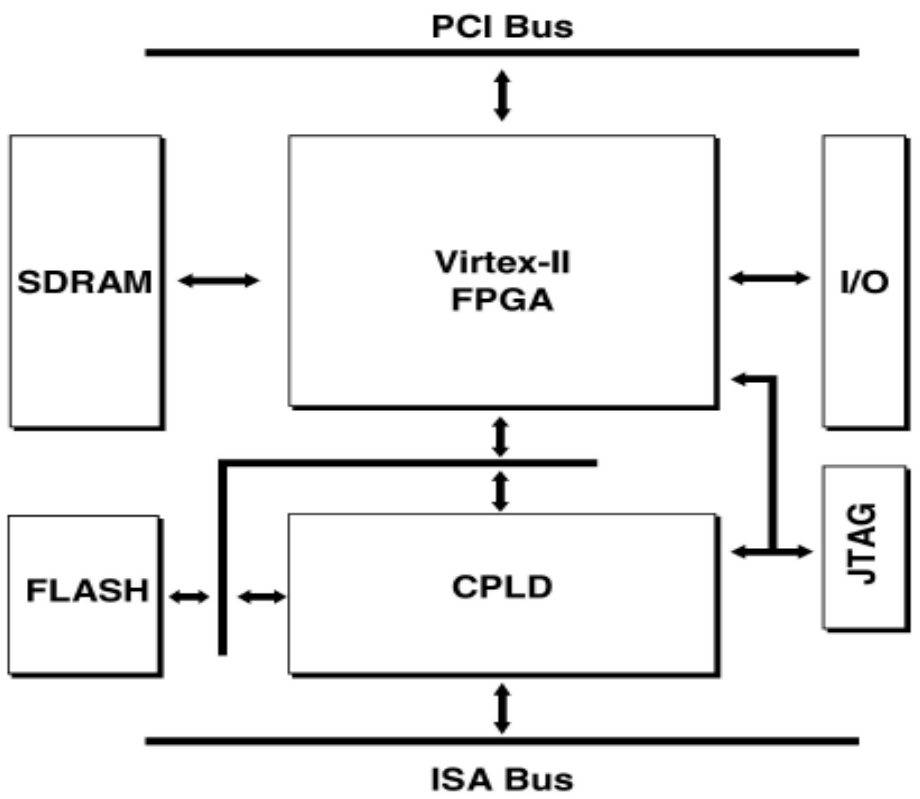

Fig. (3) PF3100 board high level block diagram 


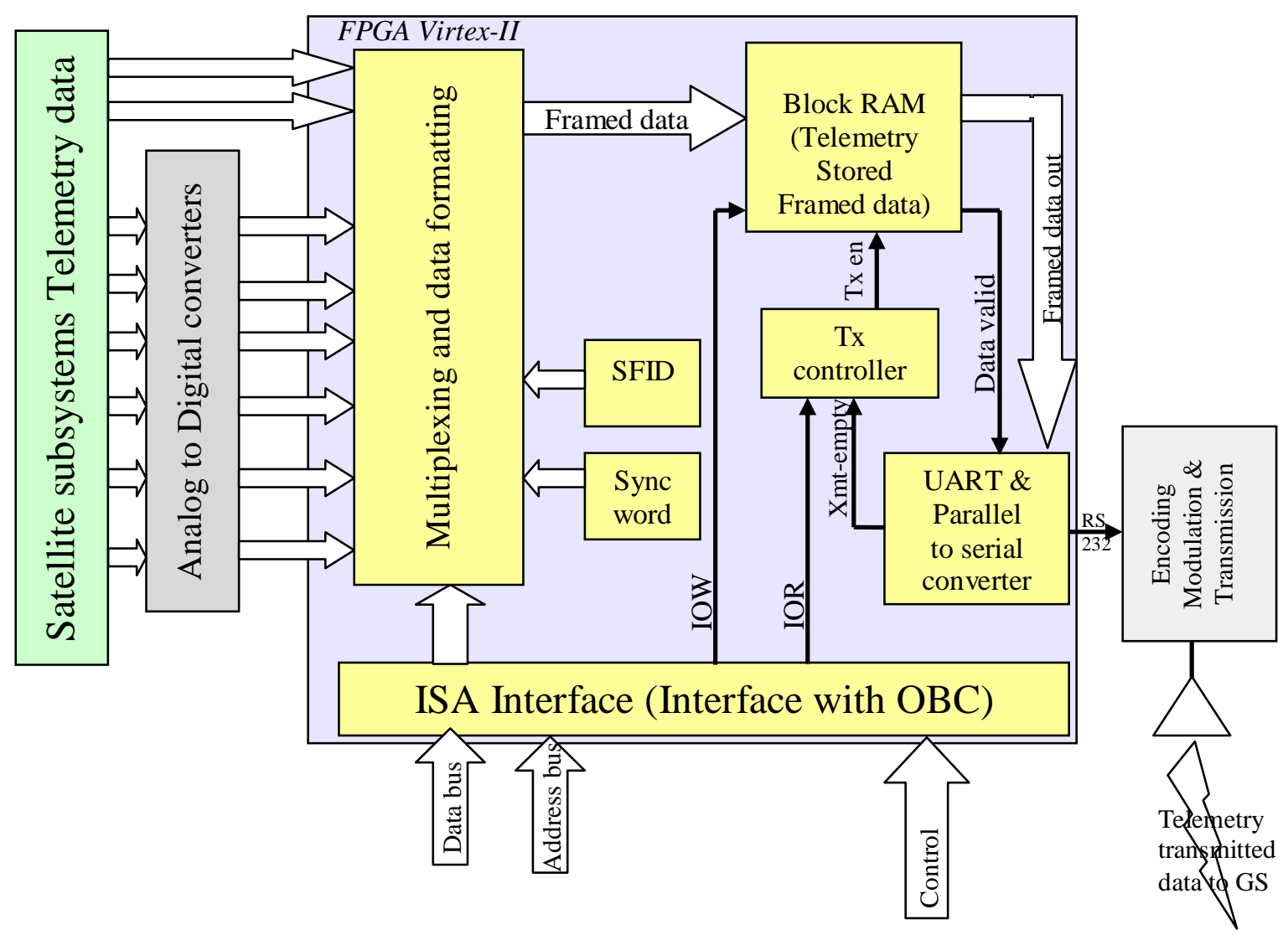

Fig. (4) Top level diagram of Telemetry board

\begin{tabular}{|l|l|l|l|l|l|l|l|l|l|l|}
\hline sync & sync & SFID & Data & Data & Data & Data & Data & Data & Data & Data \\
\hline sync & sync & SFID & Data & Data & Data & Data & Data & Data & Data & Data \\
\hline sync & sync & SFID & Data & Data & Data & Data & Data & Data & Data & Data \\
\hline sync & sync & SFID & Data & Data & Data & Data & Data & Data & Data & Data \\
\hline sync & sync & SFID & Data & Data & Data & Data & Data & Data & Data & Data \\
\hline sync & sync & SFID & Data & Data & Data & Data & Data & Data & Data & Data \\
\hline sync & sync & SFID & Data & Data & Data & Data & Data & Data & Data & Data \\
\hline sync & sync & SFID & Data & Data & Data & Data & Data & Data & Data & Data \\
\hline
\end{tabular}

Fig. (5) PCM array framed data

\section{Telecommands}

Command signals are transmitted from the GCC to the satellite to satisfy operational mission requirements or to respond to emergency conditions. Also commands may be scheduled according to the pre-known and deterministic tasks of the different satellite subsystem.

Commands may take one of the following procedures:

(1) During regular operations: a set of scheduled patch files run and execute specific functions, specific tasks, and execute specific commands. In this case the satellite in its autonomous state and only OBC govern the satellite. Each subsystem should have its own specific executable patch files to execute the required functions. 
(2) During the visibility time; the GCC may needs to command the satellite according to the analysis of the telemetry data or new assigned task. In this case the GCC should prepare new patch files instead of the old ones, these new patch files replace the old ones and are executed according to the satellite schedules.

(3) By using transceivers based on network protocols (like Aerocomm CN4790 transceivers [11]) a complete remote desktop controlling between GS and satellite can be executed.

As an illustration example of these patch files is shown in Fig. ( 6 ). It shows an example of the dot txt files, which then saved as dot bat files to be scheduled according to the satellite timing plan.

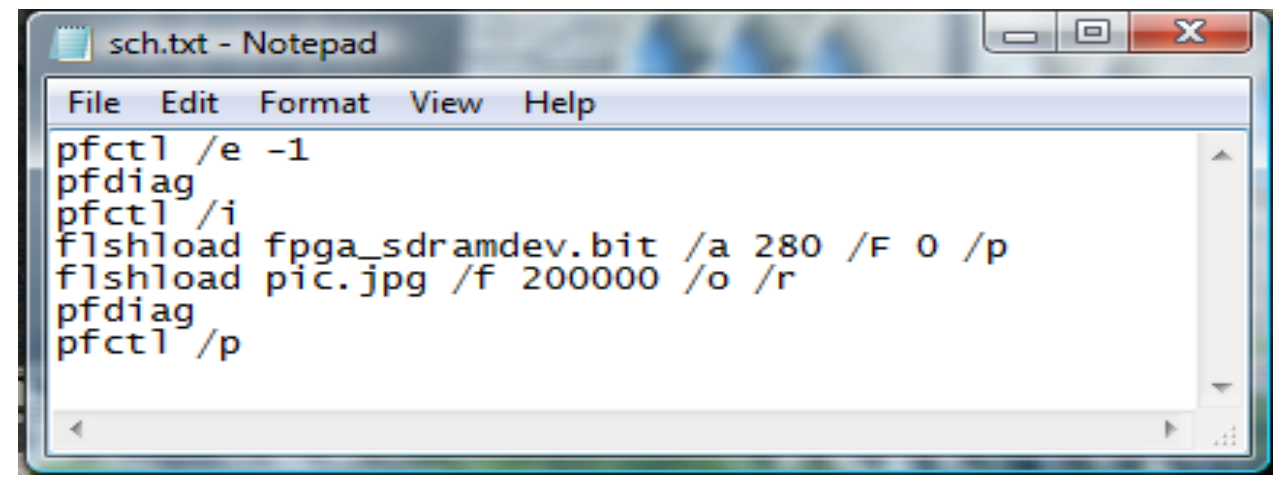

Fig. ( 6 ) Example of a prepared patch file

In this example of the command patch file, the OBC commands the telemetry board to erase all the contents of the flash memory, display a report about the telemetry board status, initialize the FPGA, save the configuration file fpga_sdramdev.bit starting on the bank zero of the Flash memory, save the pic.jpg picture starting on address 200000 of the Flash memory, again display a report about the telemetry board status, and then program the FPGA with the configuration file which saved on bank zero of the Flash memory. Simply, by this way OBC commands the telemetry board with many commands. It is also clear from this patch file that; it can contain single command line or multiple commands on the same patch file. Many patch files can be installed as components of the OS to execute assigned missions and tasks. Each subsystem on the satellite should have its own patch files to execute the required tasks. All of these patch files should organized and scheduled according to accurate satellite timing plan.

\section{Simulation and experimental results}

The preliminary engineering block diagram design of the T\&Tc subsystem is shown in Fig. (7). Mainly it consists of OBC, telemetry board, CL4790 or CN4790 transceivers, and different data buses. OBC communicates with the telemetry board via PC 104 (ISA bus) connector [4]. The Telemetry data is transmitted serially via RS 232 connector which is connected to the transceiver CL4790 and then received by the GS. The GS is emulated by a Pentium 4 PC and CL4790 or CN4790 transceivers, as shown in Fig. (8).

The FPGA configuration files were prepared on the GCC, the FPGA can be configured on the ground before flight or while the satellite is in the space. All of the control signals were taken from the ISA bus on the OBC SBC-GX1 board. Two of the main important control signals are IOW and IOR which are used in controlling the FPGA in storing the telemetry data and downlinking it, respectively. These two control signals activated with there corresponding 
storing and downlinking patch files. A simulated telemetry data are stored in the FPGA block RAM when the IOW is activated with the corresponding telemetry patch file. During the visibility period (around $10 \mathrm{~min}$ for the case study, sun synchronous orbit, $850 \mathrm{~km}$ altitude) the downlinking patch file runs, IOR becomes active, telemetry data transformed from parallel to serial, transferred to the transceiver (CL4790 or CN4790), then the telemetry data is received by the transceivers of the GS.

On the same time, satellite receives commands, patch files from the GS, respond to the new commands, and execute it after verification protocol between the satellite and GS.

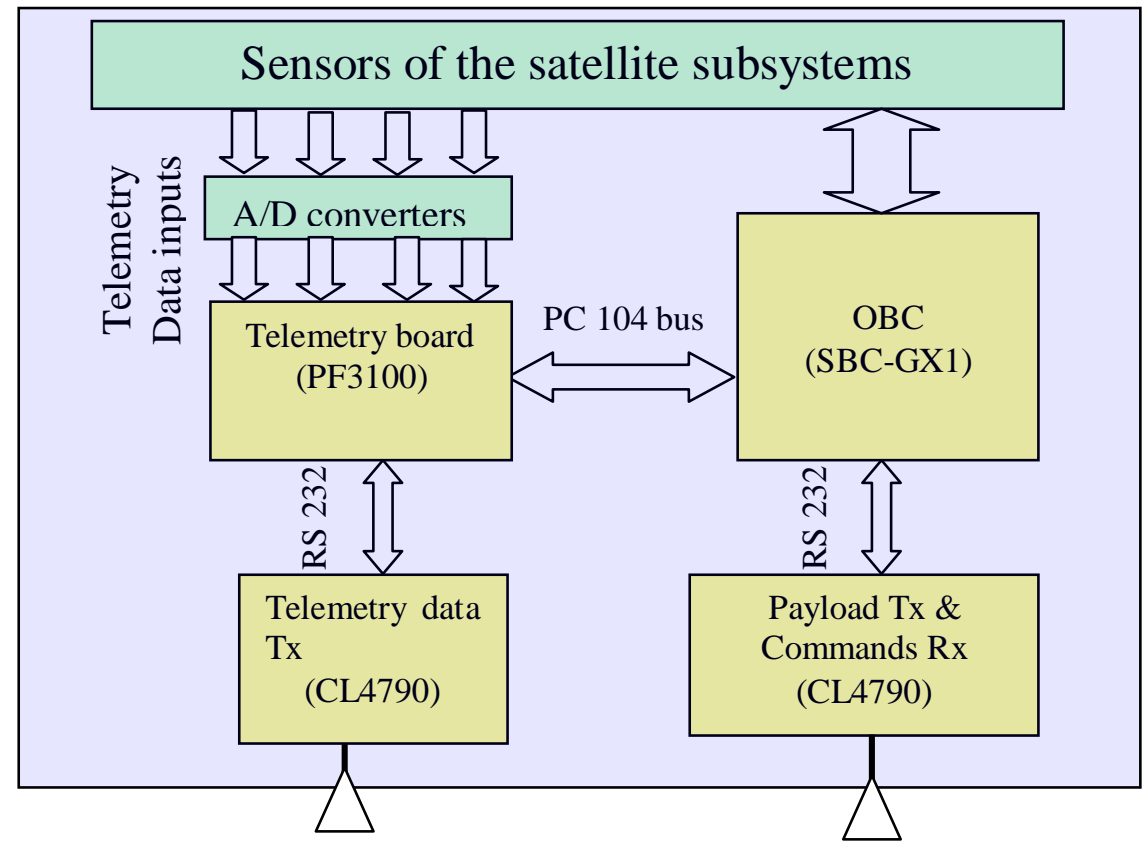

Fig. (7) Simplified block diagram of the telemetry and telecommand subsystem

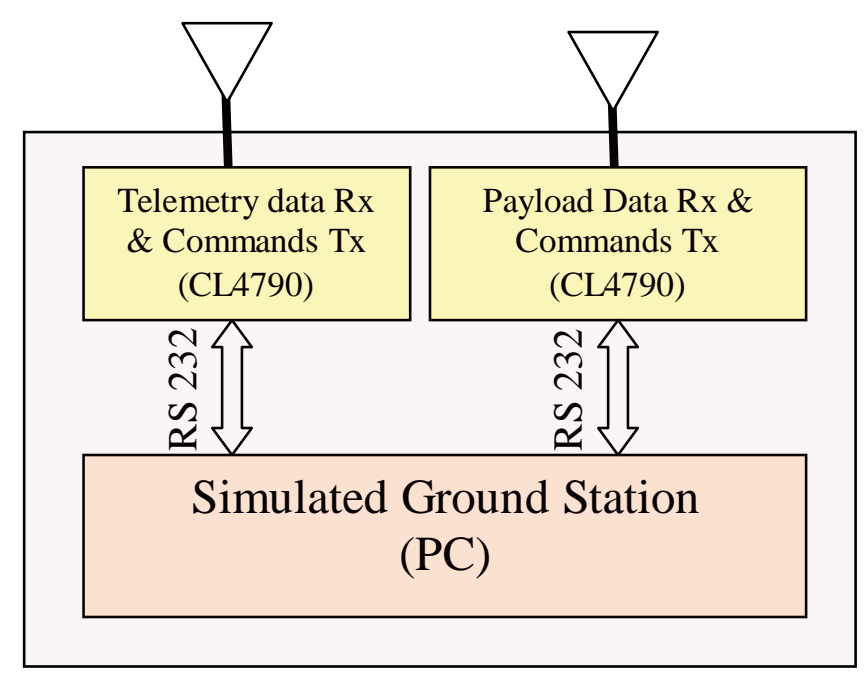

Fig. (8) Block diagram of the simulated control center ground station 


\section{Conclusion}

A hardware simulation of the telemetry and telecommand subsystem is presented. Commercial off the-shelf components are used to emulate and integrate the subsystem. Telemetry board is connected with OBC board via ISA connector, the design of the FPGA on the telemetry board is presented, the FPGA can also be reconfigured on the space. More than one configuration file can be stored on the telemetry board and loaded according to a command from the GS or OBC. Storing or downlinking of the telemetry data is executed according to specific commands and control signals from OBC. Patch files are prepared to control the telemetry and telecommand subsystem, and a new patch command files are loaded from GS to command and control the satellite. Routines run according to pre-schedules, these routines simulate the regular daily work of the satellite. Windows XP embedded is the used RTOS, it is facilitate many programming and controlling operations. Two types of transceivers are used, Aerocomm CL4790, and CN4790 with RS 232 or RJ 45 interfaces, respectively.

\section{References}

[1] John D. Jensen, "The design of the command and data handling subsystem used by the Ionospheric observation nanosatellite formation," Msc. Thesis, Utah state university, Logan, Utah, 2000.

[2] J. R. Wertz, W. J. Larson, "Space Mission Analysis and Design," $3^{\text {rd }}$ Edition, Microcosm Press, 1999.

[3] Gary D. Gordon and Walter L. Morgan, principles of Communications Satellites, John Willy \& Sons, Inc. 1993.

[4] Edward Solari, " ISA and EISA Theory and Operation," Annabooks, 2003. http://www.annabooks.com .

[5] http://www.eyassat.com .

[6] Technical manual "SBC-GX1.pdf," 2006. http://www.arcom.com.

[7] http://www.microsoft.com .

[8] Derivation system Inc., "PF3100 ${ }^{\mathrm{TM}}$ Reference manual -pdf," edition 1.5.5., July 2003. http://www.derivation.com .

[9] Technical Manual, "Xilinx ISE 9.1 Software Manuals and Help -pdf Collection", Xilinx, Inc., (2006).

[10] Stephen Horan, "Introduction to PCM Telemetring Systems", $2^{\text {nd }}$ edition, Taylor \& Francis, 2002.

[11] www.aerocomm.com . 